Check for updates

Cite this: RSC Adv., 2019, 9, 36631

Received 25th July 2019

Accepted 30th October 2019

DOI: $10.1039 / c 9 r a 05768 d$

rsc.li/rsc-advances

\section{Application of smectite for textile dyeing and fastness improvement}

\author{
Jong Sun Jung (iD a and Seong Hun Kimªb
}

Three smectite-based pigments were prepared by the intercalation of cationic dyes utilizing the ion exchangeability of smectite, and the synthesis results were verified by $\mathrm{X}$-ray diffraction and transmission electron microscopy. The study involved three fabric-dyeing methods: CD using only cationic dyes, SP using only smectite pigments, and ST using smectite post-treatment on fabrics colored with cationic dyes. For each method, dyeability, washing fastness, and colorfastness to artificial-light exposure were assessed by the absorption-to-scattering ratio $(K / S), \Delta K / S$, and changes in color difference. Overall, $C D$ showed high dyeability but low washing fastness and light fastness for all fabrics. In contrast, SP showed low dyeability and small changes in K/S and changes in color difference, representing washing fastness and light fastness, respectively. ST showed good dyeability, indicating that smectite post-treatment had improved both washing fastness and light fastness. Based on these findings, the improved fastness was attributed to dye release from the fabrics colored with the cationic dye during smectite post-treatment, as the dye simultaneously entered the interlayer space between the smectite layers, while coating the fabric surface with smectite.

\section{Introduction}

Since the development of synthetic dyes, the field has grown rapidly and has become the mainstay of textile coloring owing to their diverse range of colors, the ease of dyeing, and excellent fastness. However, synthetic dyes are causing many problems for the environment and humans owing to the hazardous materials produced during dye manufacture, preparation, and handling and to wastewater produced during dyeing: ${ }^{1-5}$ When natural dyes are used, chemical (i.e., metallic) mordants such as $\mathrm{Cu}^{2+}$ and $\mathrm{Cr}^{6+}$ are used to increase dyeability and fastness. As a result, such mordants accumulate over a long time without readily dissolving in soil and water, which may retard crop growth and have harmful effects on human health. ${ }^{6,7}$ Accordingly, recent studies have used more environmentally friendly natural materials such as Al, ${ }^{8} \mathrm{Mg},{ }^{9}$ chitosan, ${ }^{10}$ tannin,${ }^{11,12}$ alum, and bittern to enhance dyeability, fastness, and functionality. ${ }^{13}$

In one previous study, colored natural clay minerals were used to color fabrics firmly without mordants, and dyeability was superior when clay particles were smaller. In particular, fine particles had spread into the fiber interior, thereby improving washing fastness. ${ }^{14,15}$ However, natural clay minerals only offer a limited array of colors, and it is often difficult to find and reproduce materials with the exact color. The present study

${ }^{a}$ The Research Institute of Industrial Science, Hanyang University, Seoul 04763, Republic of Korea. E-mail: kimsh@hanyang.ac.kr

${ }^{b}$ Department of Organic and Nano Engineering, Hanyang University, Seoul 04763, Republic of Korea investigated methods of improving dyeability and fastness using smectite among other clay minerals because it consists of fine particles and could produce a wide variety of colors by dye intercalation. Smectite already is being used as an adsorbent, rheology-controlling agent, ion exchanger, catalyst, and catalyst support in various fields including civil engineering, paints, and cosmetics. ${ }^{16}$ Meanwhile, studies on smectite and dyes have reported application to ink pigments ${ }^{17}$ and dye-wastewater treatment. ${ }^{18,19}$

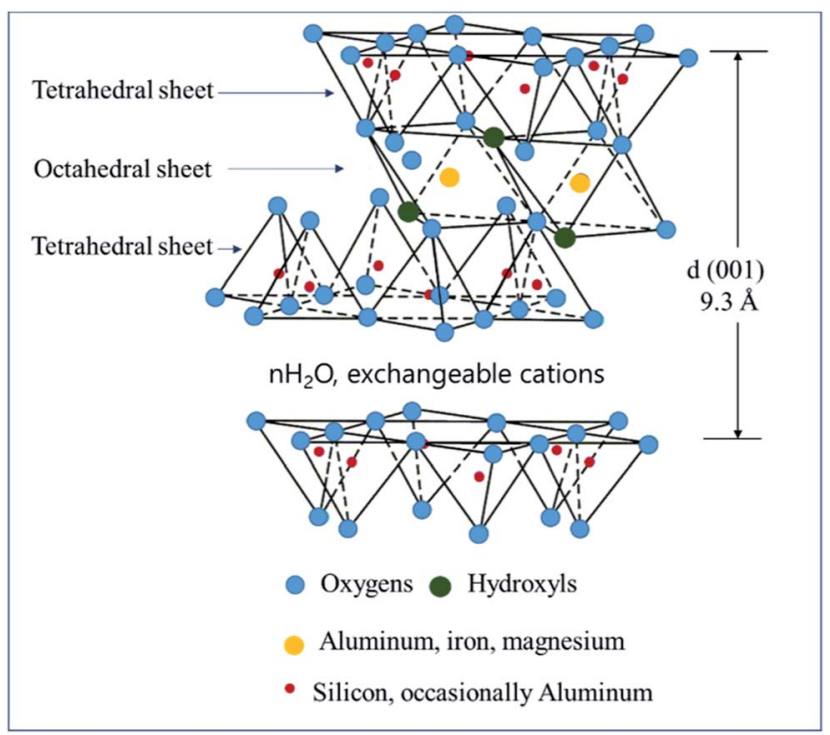

Scheme 1 Smectite structure. ${ }^{20}$ 
Smectite is the name used for a group of phyllosilicate mineral species, and as shown in Scheme 1, it has a chemical composition of $\mathrm{T}_{2} \mathrm{O}_{5}(\mathrm{~T}=\mathrm{Si}, \mathrm{Al}, \mathrm{Be})$ and continuous twodimensional tetrahedral sheets. Three of the four vertices of these tetrahedra are shared with other tetrahedra, whereas the fourth vertex point is in a direction different form the other vertices while remaining unshared. It is also known as a $2: 1$ clay wherein tetrahedral sheets are bonded (or coordinated) to octahedral ones or to independent cations. ${ }^{21,22}$ Smectite shows ultrafine grains and wide surface area as well as expansion in water, ion exchangeability, surface-current-induced deformation of exchangeable ions, and interaction with inorganic and organic liquids. ${ }^{23}$ Among these properties, the present study utilized smectite that allows the adsorption of cations and anions because of the applied surface current and the retention of exchangeable ions between layers or on the surface, where these ions could be exchanged for cations or anions in aqueous solutions. When clay minerals are mixed in aqueous ionic solutions above a certain concentration, ion exchange occurs. By measuring concentrations of cations or anions released into the solution during ion exchange, negative and positive charges of clay minerals could be determined. Herein, the concentration of cations participating in the reaction implies the cation exchange capacity (CEC). Typically, clay minerals showing charged layers show relatively high CEC values. Smectite is known to show CEC values in the range $60-150 \mathrm{mEq} / 100 \mathrm{~g} .{ }^{23-25}$

Although smectite is used in various fields, there have been almost no studies on using smectite for textile dyeing. In the present study, the ion exchangeability of smectite was utilized to prepare pigments for textile dyeing by the intercalation of variously colored cationic dyes. Moreover, smectite post-treatment was applied to dyed fabrics to improve the dyeability, washing fastness, light fastness. The objective was to find a more environmentally friendly coloring or dyeing method that uses only smectite and no mordant. The study investigated the coloring mechanism by component and structural analyses to identify the effect of smectite during coloring and post-treatment. The findings of the present study suggest that smectite could be used to improve the fastness of plant-based natural dyes, which typically show low fastness owing to discoloration and fading due to lightinduced chemical reactions.

\section{Materials and methods}

\subsection{Materials}

Smectite samples obtained from Kurosaki Hakudo Industry Co., Ltd. in Japan, had a mean particle size of $0.23 \mu \mathrm{m}$ and CEC of 68 $\mathrm{mEq} / 100 \mathrm{~g}$. The element compositions of the obtained smectite
Table 2 Fiber characteristics of $100 \%$ cotton, silk, and wool test fabrics

\begin{tabular}{lllll}
\hline & $\begin{array}{l}\text { Weight }(\mathrm{g} \\
\left.\mathrm{m}^{-2}\right)\end{array}$ & $\begin{array}{l}\text { Density: warp } \times \\
\text { weft }\left(5 \mathrm{~cm}^{2}\right)\end{array}$ & $\begin{array}{l}\text { Thickness } \\
(\mathrm{mm})\end{array}$ & Weave \\
\hline Cotton & 126.5 & $116.2 \times 106.4$ & 0.32 & Plain \\
Silk & 50.5 & $240.2 \times 212.6$ & 0.13 & Plain \\
Wool & 102 & $72 \times 69$ & 0.25 & Plain
\end{tabular}

are shown in Table $1 . \mathrm{SiO}_{2}$ and $\mathrm{Al}_{2} \mathrm{O}_{3}$, which are commonly found in silicate minerals, were the principal components, while $\mathrm{Fe}_{2} \mathrm{O}_{3}, \mathrm{MgO}, \mathrm{CaO}, \mathrm{Na}_{2} \mathrm{O}, \mathrm{K}_{2} \mathrm{O}$, and other oxides were minor components.

Rhodamine B, Berberine chloride $n$-hydrate, and methylene blue trihydrate (Wako Chemical, special reagent grade) were used for red, yellow, and blue cationic dyes, respectively.

Test fabrics consisted of $100 \%$ cotton, silk, and wool, and the corresponding fiber characteristics are shown in Table 2.

\subsection{Preparation of smectite pigments by cationic-dye intercalation}

Liquid A, a smectite suspension, was prepared by adding $215 \mathrm{~g}$ of water to $85 \mathrm{~g}$ of smectite slurry. For liquid B, $10 \mathrm{mmol}$ of each dye was dissolved in $200 \mathrm{~g}$ of deionized water at $70^{\circ} \mathrm{C}$. Liquid $\mathrm{B}$ was slowly dropped into liquid A at $70{ }^{\circ} \mathrm{C}$ for $30 \mathrm{~min}$ and then the reactants were stirred at $300 \mathrm{rpm}$ for $1 \mathrm{~h}$ at $70{ }^{\circ} \mathrm{C}$. The obtained substance was filtered using cellulose paper. The precipitate was washed twice in the range $50-70{ }^{\circ} \mathrm{C}$ and subsequently washed thrice at room temperature with $100 \mathrm{~mL}$ of water each time. The substance was dried for $24 \mathrm{~h}$ at $35{ }^{\circ} \mathrm{C}$ and then crushed in a mortar.

\subsection{Methods of coloring and finishing fabric}

For $\mathrm{CD}$, all the fabrics were dyed at $80{ }^{\circ} \mathrm{C}$ for $60 \mathrm{~min}$ while being agitated at $100 \mathrm{rpm}$ in each of the three cationic dyes prepared in a concentration of $2 \%$ weight of fiber (W.O.F.) and a liquor ratio of $1: 50$.

For SP, all the fabrics were dyed at $80{ }^{\circ} \mathrm{C}$ for $60 \mathrm{~min}$ while being agitated at $100 \mathrm{rpm}$ in each of the 3 smectite pigments prepared in a concentration of $2 \%$ weight of fiber (W.O.F.) and a liquor ratio of $1: 50$.

For ST, all the fabrics initially were dyed with each cationic dye under the same conditions as those for $\mathrm{CD}$ and were subsequently treated with smectite. The dyed fabric specimens $(1 \mathrm{~g})$ were agitated in $100 \mathrm{~g}$ of liquid $\mathrm{A}$ at $70{ }^{\circ} \mathrm{C}$ for $30 \mathrm{~min}$ and subsequently rinsed thrice with $100 \mathrm{~mL}$ of water each time.

Table 1 Element compositions of smectite as determined by semiquantitative X-ray (SQX) fluorescence analysis

\begin{tabular}{|c|c|c|c|c|c|c|c|c|}
\hline & $\mathrm{SiO}_{2}$ & $\mathrm{Al}_{2} \mathrm{O}_{3}$ & $\mathrm{Fe}_{2} \mathrm{O}_{3}$ & $\mathrm{MgO}$ & $\mathrm{CaO}$ & $\mathrm{Na}_{2} \mathrm{O}$ & $\mathrm{K}_{2} \mathrm{O}$ & Others \\
\hline Chemical composition (\%) & $M=60.09$ & $M=101.95$ & $M=159.79$ & $M=40.32$ & $M=56.08$ & $M=61.99$ & $M=94.20$ & - \\
\hline
\end{tabular}




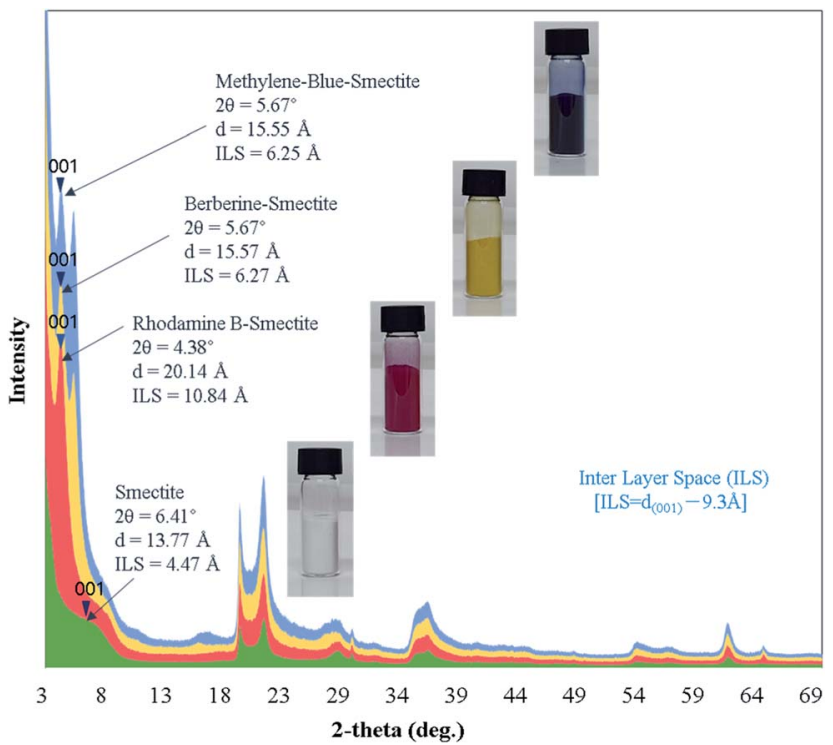

Fig. 1 XRD profiles of smectite and smectite pigments.

\subsection{Characterization}

Elemental analyses of smectite were performed by semiquantitative X-ray fluorescence (SQX, ZSX Primus П, Rigaku
Co., Ltd.) and high-resolution X-ray diffraction (HR-XRD, powder X-ray diffractometer, Smart Lab, Rigaku Co., Ltd.).

Smectite interlayer space (ILS) was calculated by eqn (1), as follows:

$$
\mathrm{ILS}=d(001)-9.3 \AA
$$

where $d(001)$ is the measured thickness of a smectite layer with dye, and $9.3 \AA$ is the theoretical thickness of a smectite layer without dye.

The crystal structure was analyzed using transmission electron microscopy (TEM, TEM-2100F, JEOL, Ltd.), while permeability was measured using ultraviolet-visible (UV-vis) spectrophotometry (V-670, JASCO).

Fabric color-yield was determined by the absorption-toscattering ratio $(K / S)$ calculated by the Kubelka-Munk equation from the average reflectance spectra of the colored fabrics, as measured in the range $400-700 \mathrm{~nm}$ by a JS-555 spectrum color meter (Shimadzu UV-240). The absorption-to-scattering ratio is given by eqn (2), as follows:

$$
K / S=(1-R)^{2} / 2 R,
$$

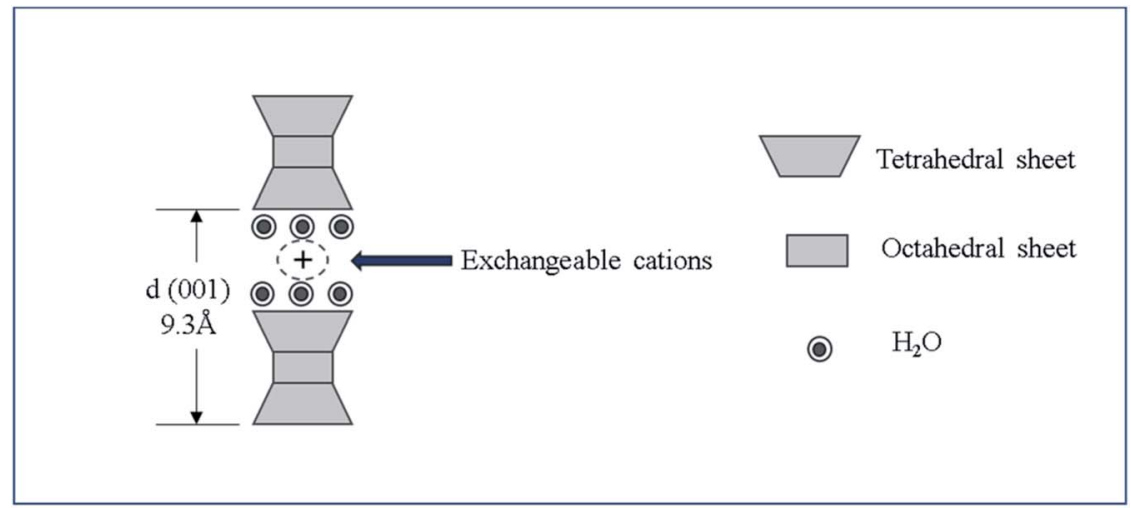

Scheme 2 Smectite interlayer structure showing the interlayer space.
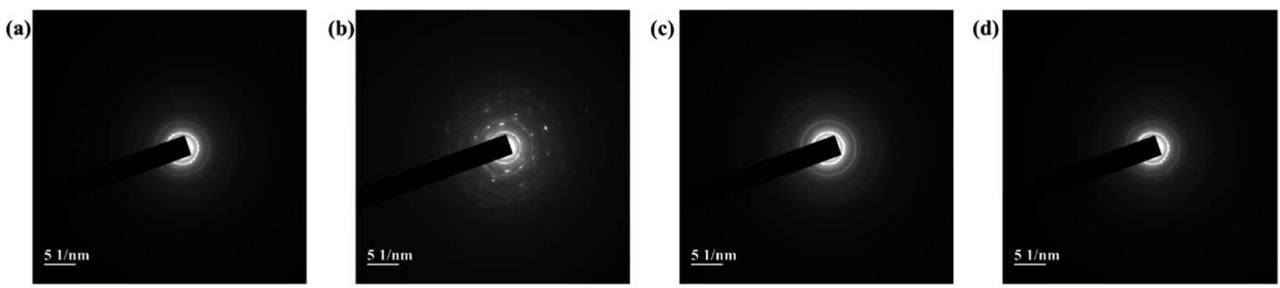

(e)
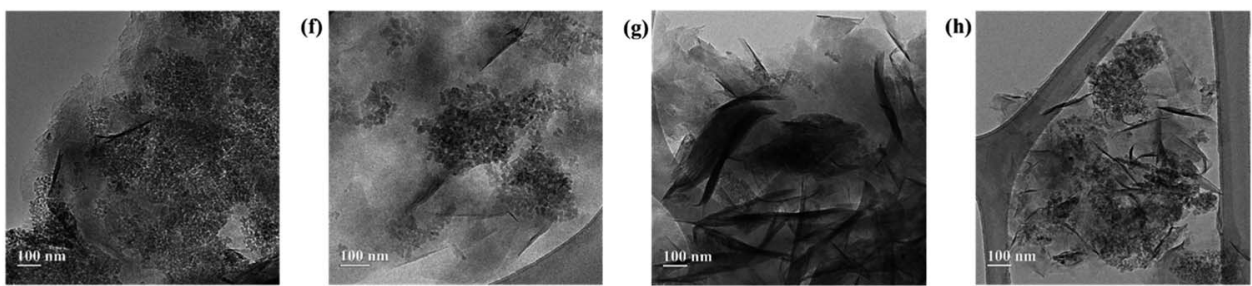

Fig. 2 TEM images of (a), (e) pure smectite and (b), (f) rhodamine B-; (c), (g) berberine chloride-; and (d), (h) methylene blue-smectite pigments. 
where $K, S$, and $R$ are the absorption, scattering, and reflection coefficients, respectively.

$L^{*} a^{*} b^{*}$ (CIE 1976) chromaticities were determined using NF777.

Washing tests were carried out according to ISO 105-C06:2014 Textiles-Tests for Colorfastness-Part C06: Colorfastness to domestic and commercial laundering at $30 \pm 2{ }^{\circ} \mathrm{C}$ for $30 \mathrm{~min}$.

Light exposure test was carried out according to ISO 105B02:2014 Textiles-Tests for Color fastness-Part B02: Color fastness to artificial light: Xenon arc fading lamp test.

\section{Results and discussion}

\subsection{Confirmation of smectite pigment intercalation by XRD analysis}

The XRD profiles of smectite and smectite pigments prepared by the intercalation of each guest dye into smectite (host) are shown in Fig. 1. ILSs of smectite and the smectite pigments were calculated using eqn (1). The theoretical interlayer distance is 9.3 $\AA$, as shown in Scheme 2. The ILSs of smectite, rhodamine Bsmectite, Berberine-smectite, and methylene blue-smectite were $4.47,10.84,5.67$, and $6.25 \AA$, respectively. Because ILS represents the thickness of space occupied by molecules intercalated into the layered smectite structure, ILS $=4.47 \AA$ in the pure smectite sample (without any dye) represents the thickness of the space occupied by $\mathrm{H}_{2} \mathrm{O}$ molecules. Therefore, the ILSs of smectite pigments, ranging between 5.67 and $10.84 \AA$, represented the thickness of the spaces created and occupied by molecules of the cationic dyes. Accordingly, the ILS results confirmed intercalation of cationic dyes into smectite. Moreover, it was visually confirmed that smectite did not cause colors to deviate significantly from those of the existing aqueous cationic dye solutions.

\subsection{Confirmation of smectite pigment intercalation by TEM analysis}

TEM images of the smectite and the smectite pigments are shown in Fig. 2, wherein (a), (b), (c), and (d) are the patterns of electronic diffraction that appear to cause scattering when the beam passes through a constant spacing. Diffraction rings appeared in (a), (c), and (d) owing to electron scattering in irregularly coordinated polycrystals; whereas, in (b), a diffraction ring representing polycrystals and diffraction points representing monocrystalline diffraction by reciprocal lattices appeared together. Smectite minerals are usually monocrystals showing very diverse facies. However, most particles are small in size, and the particle boundaries are unclear. The diffraction rings appeared very thin, indicating irregular deposition and aggregate formation. ${ }^{26}$ TEM images (e), (f), (g), and (h) show that phyllosilicate minerals have

(a)

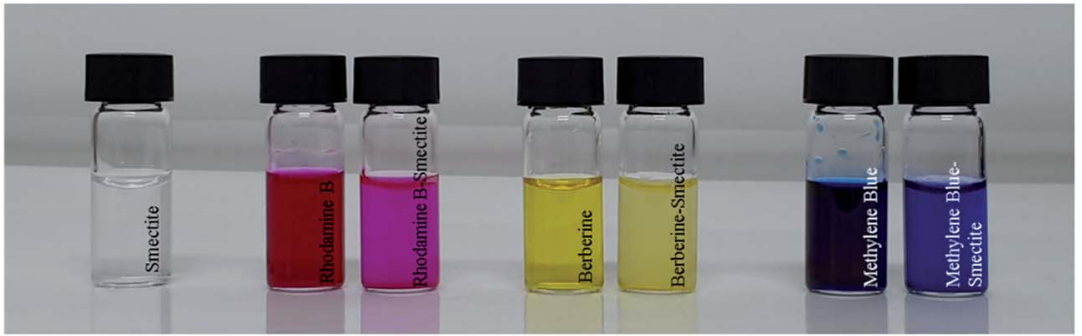

(b)

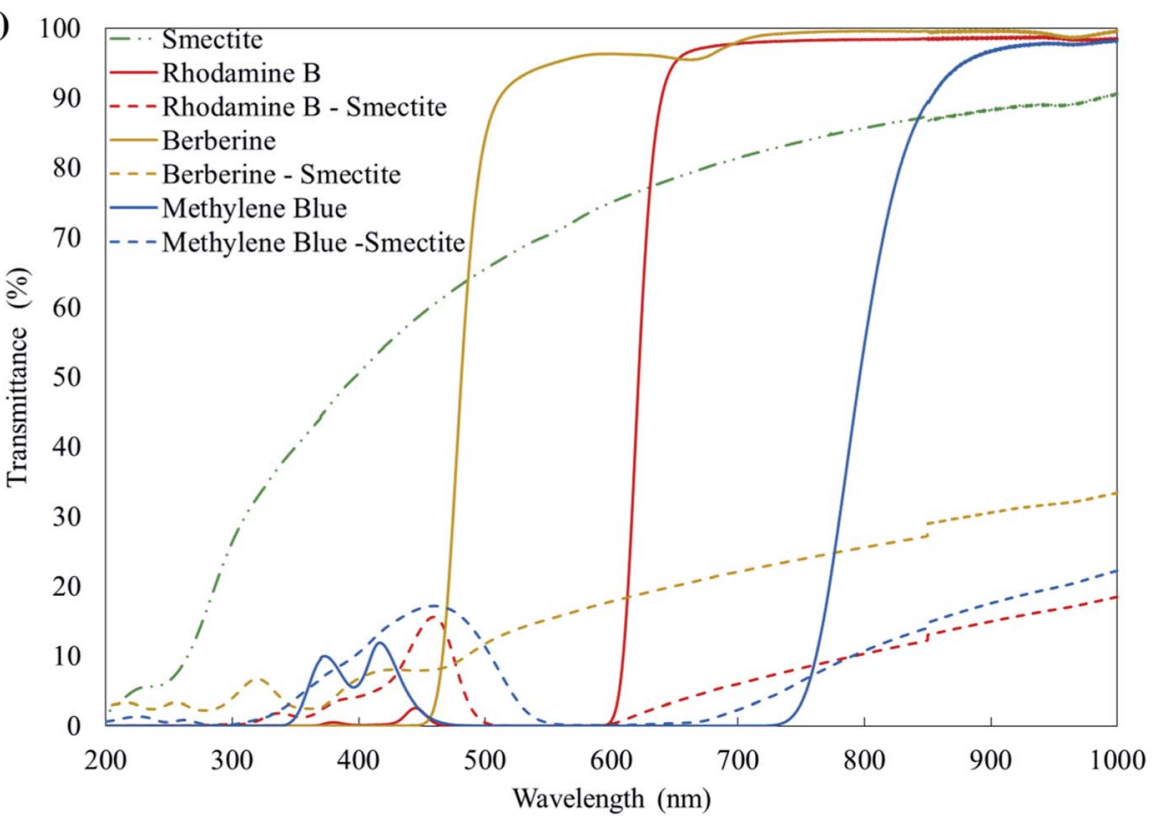

Fig. 3 Transmittances of smectite, dyestuffs, and smectite pigments: (a) pictures and (b) corresponding UV-vis transmittance spectra. 
thin plate-like crystals that have a laminated structure. In the comparison between smectite (e) and smectite pigments prepared with intercalation of cationic dyes [(f), (g), and (h)], $\mathrm{Mg}^{2+}, \mathrm{Ca}^{2+}$, $\mathrm{Na}^{+}$, and $\mathrm{K}^{+}$present in the ILS had escaped via ion exchange and the cationic dye occupied the space previously occupied by those ions. Ion exchange by the destruction of the bonding structure (i.e., reaction at the side surface of particles) is usually $<20 \%$ in smectite, while most could be attributed to lattice substitution (i.e., reaction at the bottom surface). Moreover, smectite capable of interlayer expansion is known to have approximately $80 \%$ exchange reaction on the bottom surface, with the rest occurring at the ridge.

\subsection{Dispersibility and permeability of smectite, dyestuff, and smectite pigments}

The transmittances of smectite, dyestuffs, and smectite pigments are shown in Fig. 3. The transparency of aqueous solutions, which corresponded to the dyeing conditions used for the smectite, dyestuffs, and smectite pigments, were measured by UV-vis spectrophotometry. Visually, the colors of dyestuff solutions appeared deeper than those of corresponding smectite pigment solutions, which was also confirmed by UVVis transmittance measurements. Rhodamine $\mathrm{B}$, berberine, and methylene blue solutions, indicated by solid lines, showed transmittances of approximately $90 \%$ in the maximum visible spectrum range 380-800 $\mathrm{nm}$, while smectite did not disperse very well, showing suspended particles without much sedimentation. Meanwhile, smectite pigments, indicated by dotted lines, showed transmittances of $\leq 20 \%$, indicating that the particles had formed aggregates. ${ }^{27}$

\section{4. $\quad K / S$ spectra and the corresponding $L^{*}, a^{*}$, and $b^{*}$ of cotton fabric colored with cationic dyes by $\mathrm{CD}, \mathrm{SP}$, and ST}

The $K / S$ spectra and the corresponding $L^{*}, a^{*}$, and $b^{*}$ values of cotton fabrics dyed by CD, SP, and ST are shown in Fig. 4. Basic dyes (i.e., cationic dyes) can be used directly on wool, silk, and nylon in a neutral or slightly acidic bath solution. However, it is difficult to directly color cellulose textiles such as cotton and rayon because they usually do not show an affinity for cationic dyes. Thus, such textiles are colored by mordanting. Smectite was used on cotton fabric, which showed lowest dyeability, to determine whether smectite could improve dyeability. The highest $K / S$ was achieved when CD was used. The $K / S$ of ST value was approximately half that of $\mathrm{CD}$, while SP showed the lowest dyeability. In other words, dyeability followed the order CD $>$ ST $>$ SP. In (d)-(i), although $L^{*}, a^{*}$, and $b^{*}$ varied according to the type of dyes used, SP showed the highest $L^{*}$ (brightness), low $a^{*}$ (red-green), and the lowest $b^{*}$ (yellow-blue) overall. Such results indicated the same tendency as the dyeability obtained from the $K / S$ spectra. Therefore, although smectite pigments alone showed poor textile dyeability, applying ST led to produce higher dyeability than that with SP. However, a previous study ${ }^{\mathbf{1 4}}$ reported that deep-color dyeing of cotton fabric was possible through repeated dyeing, where deeper color was achieved with

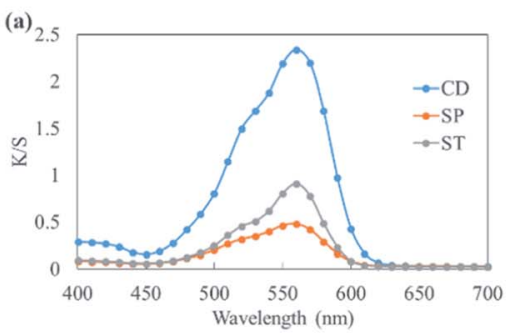

(d)

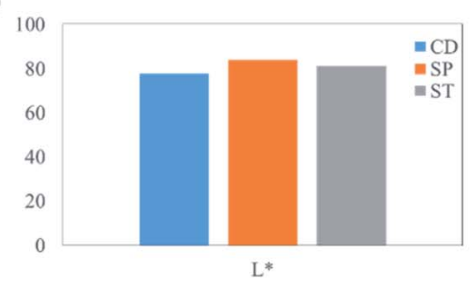

(g)

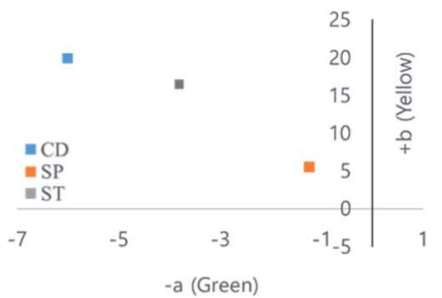

(b)

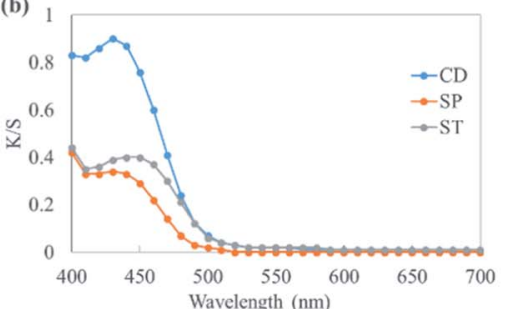

(e)

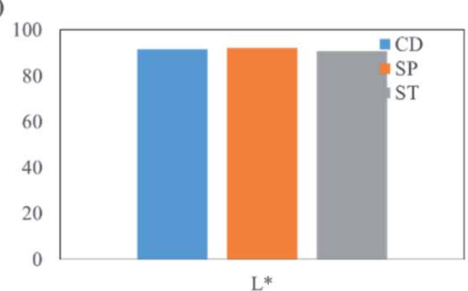

(h)

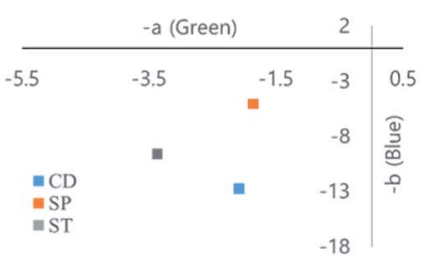

(c)

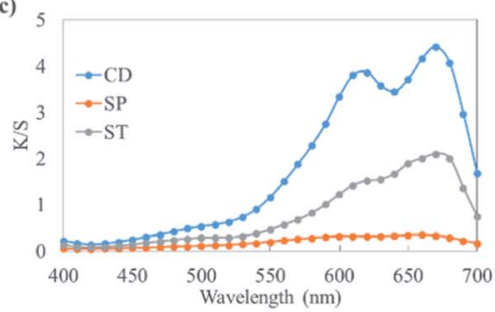

(f)
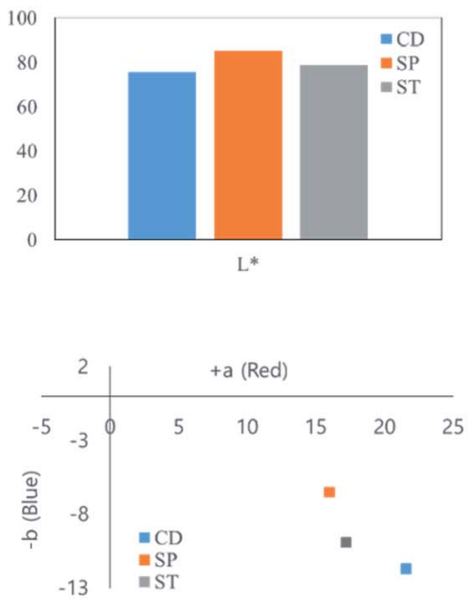

Fig. $4 \mathrm{~K} / \mathrm{S}$ spectra and corresponding $L^{*}, a^{*}$, and $b^{*}$ of cotton fabric colored with cationic dyes by CD, SP, and ST: (a), (d), (g) rhodamine B; (b), (e), (h) berberine chloride $n$-hydrate; and (c), (f), (i) methylene blue trihydrate. 

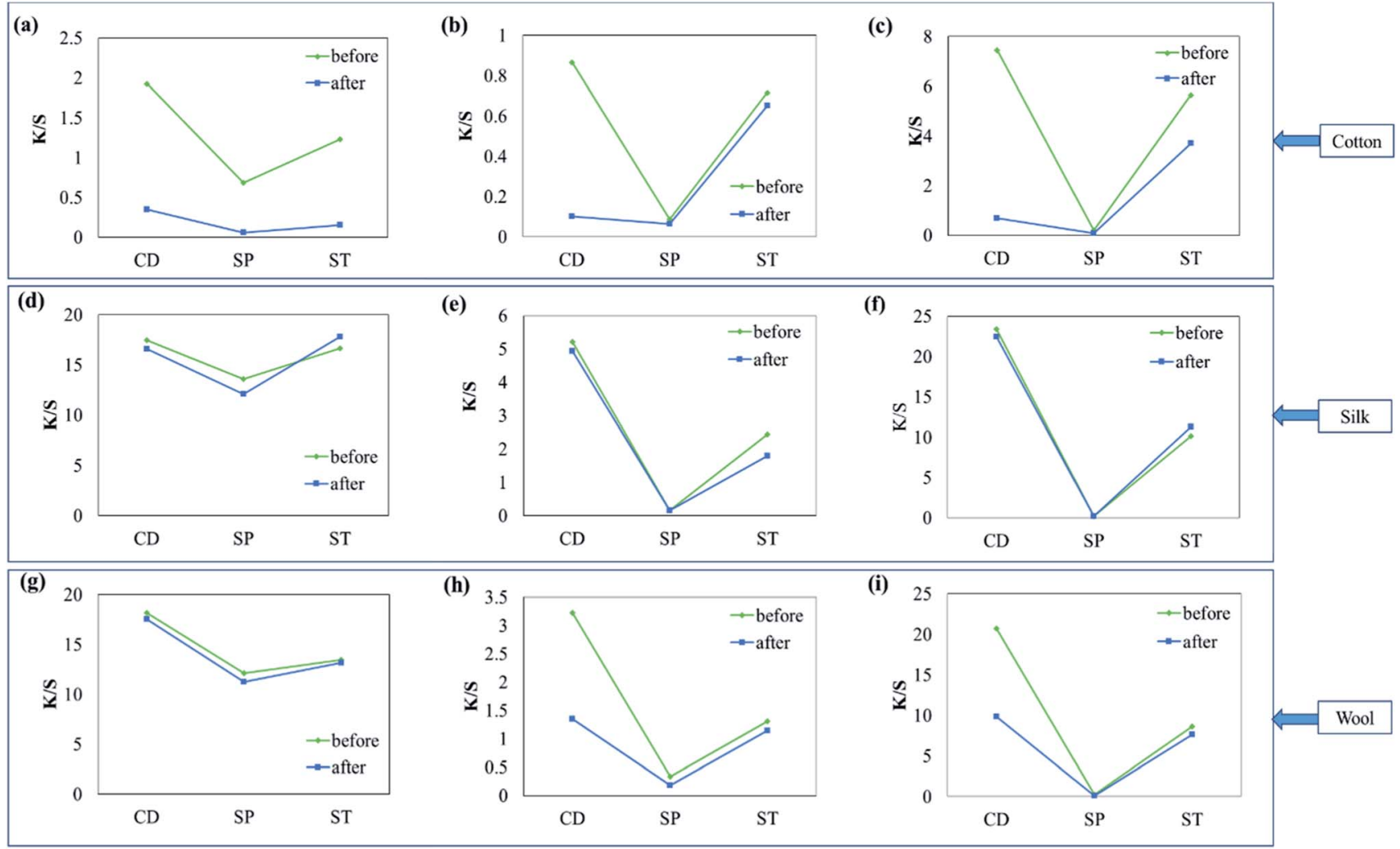

Fig. 5 Effects of CD, SP, and ST on fabric colorfastness before and after washing of cotton, silk, and wool fabrics colored with cationic dyes: (a), (d), (g) rhodamine B; (b), (e), (h) berberine chloride $n$-hydrate; and (c), (f), (i) methylene blue trihydrate.

smaller clay particles. Therefore, it is believed that dyeability could be increased by combining smectite pigments with various post-treatments.

\subsection{Characteristics of coloring and washing resistance achieved by CD, SP, and ST}

The results of washing fastness assessed by $K / S$ obtained before and after washing the fabrics dyed using the different dyes relative to the peak $K / S$ obtained for the cotton fabric dyed by $\mathrm{CD}$ are shown in Fig. 5. Although cotton samples dyed with different dyes, as shown in (a)-(c), showed the highest $K / S$ with $\mathrm{CD}, K / S$ drastically decreased after washing. These results demonstrated that cellulose textiles usually do not have any affinity for cationic dyes. As a result, almost all of the dyes had escaped from the fabric after only a single wash, indicating low washing fastness. When the fabrics were dyed using smectite pigments (SP), the coloration was too low to assess the washing fastness. When smectite post-treatment was applied to the fabrics colored with cationic dyes (ST), the cotton fabric showed lower dyeability than that did when using $\mathrm{CD}$, but $K / S$ obtained after washing showed a smaller degree of change as compared to that in $\mathrm{CD}$ for all three dyes. These results indicated improved washing fastness.

Silk samples, as shown in (d)-(f), showed good dyeability and washing fastness when dyed with CD. When ST was applied, the dyeability was lower than that achieved in CD, but there was little change between $K / S$ obtained before and after washing, indicating good washing fastness.

Cationic dyes form salt linkages with wool fibers and are mostly used for dyeing the fabric with bright colors. However, because of their low colorfastness these dyes are rarely used for dyeing wool. $K / S$ decreased by approximately one-half of the initial value after washing in $\mathrm{CD}$, except in the case of $(\mathrm{g})$. Similar to the other fabrics, wool samples post-treated using smectite showed good washing fastness, as indicated by the little change between $K / S$ obtained before and after washing. Although different fabrics showed different degrees of improvement in dyeability and fastness, ST showed greater improvement in washing fastness than did CD without smectite post-treatment. In particular, ST was effective for improving dyeability and washing fastness in cellulose textiles such as cotton, which have no affinity for cationic dyes.

\subsection{Light fastness of finished fabrics achieved by CD, SP, and ST}

The evaluation of lightfastness is shown in Table 3 as a color change class. In general, cationic dyes appeared to be mostly poorer in grades in all fabrics dyed using CDs, as they are poorly resistant to daylight. In the case of berberine in the cotton sample, the SP was the highest grade (8), following the order SP $>\mathrm{ST}>\mathrm{CD}$. In the case of rhodamine $\mathrm{B}$ and methylene blue, SP and ST had the same rating. However, the lightfastness in the case of rhodamine $\mathrm{B}$ did not improve notably because of 
Table 3 Lightfastness color change rating of fabrics colored with cationic dyes by CD, SP, and ST

\begin{tabular}{lllll}
\hline \multirow{5}{*}{ Cotton } & & \multicolumn{2}{l}{ Lightfastness (ISO 105-B02:2014) } \\
\cline { 3 - 5 } & & Rhodamine B & Berberine & Methylene Blue \\
\hline \multirow{5}{*}{ Silk } & CD & 1 & 2 & 1 \\
& SP & 2 & 7 & 5 \\
& ST & 1 & 3 & 5 \\
& CD & 1 & 1 & 1 \\
& SP & 3 & 4 & 4 \\
& ST & 3 & 2 & 4 \\
& CD & 1 & 4 & 1 \\
& SP & 4 & 6 & 4 \\
& ST & 3 & 5 & 4
\end{tabular}

smectite, as the red chromophore was weakened by the ultraviolet rays of the sun and the chemical structure was ruptured. Similar trends were also seen for silk and wool samples. In other words, SP and ST were more effective for improving light fastness than CD for all fabrics. Usually, when cotton fibers are exposed to sunlight for a long period, yellowing and gradual fiber embrittlement may occur. Silk is exposed to sunlight loses its strength because of the degradation caused by ultraviolet rays, while degradation and yellowing may also occur because of oxygen in the air. However, SPs, prepared by smectite-dye intercalation, were the most effective for improving light fastness because smectite minerals reflect ultraviolet sunlight rays. It is suspected that ST showed lower light fastness than did SPs because ultraviolet rays were reflected by the areas covered with smectite due to discoloration of the dyed fabrics. Accordingly, it is believed that using smectite pigment or adsorbing/ adhering as much smectite as possible on fabrics during posttreatment would be effective for improving light fastness.

\subsection{Mechanism underlying for textile dyeing using ST}

As shown in Fig. 1 and 2, the intercalation of cationic dyes into the smectite ILS was confirmed. To investigate the mechanism of the adsorption/adhesion on fabrics dyed using SP and ST, cotton fabric samples dyed with SP and ST were analyzed by XRD (Table 4). First, when ILS was measured at 90\% relative humidity (RH) after the cotton samples were dried for $60 \mathrm{~h}$, the same manufacturing conditions as those for the smectite pigments, the results showed that ILS $=0 \AA$. When ST was applied to nondyed cotton fabric, ILS $=4.0 \AA$, which was suspected to represent ILS because of $\mathrm{H}_{2} \mathrm{O}$ molecules. Overall, when SP was used for adsorption/adhesion on fabrics, there were no major differences in the ILS as compared with that in case of the smectite pigment before adhesion. However, for adhesion on fabrics using ST, the smectite ILS became narrower for each dye. Moreover, because the dip method was used during dyeing, measurements were carried out after evaporating as much $\mathrm{H}_{2} \mathrm{O}$ as possible from the smectite ILS at $150^{\circ} \mathrm{C}$ to eliminate the influence of $\mathrm{H}_{2} \mathrm{O}$ on the ILS. The results

Table 4 Changes in smectite ILS achieved by different dyeing methods

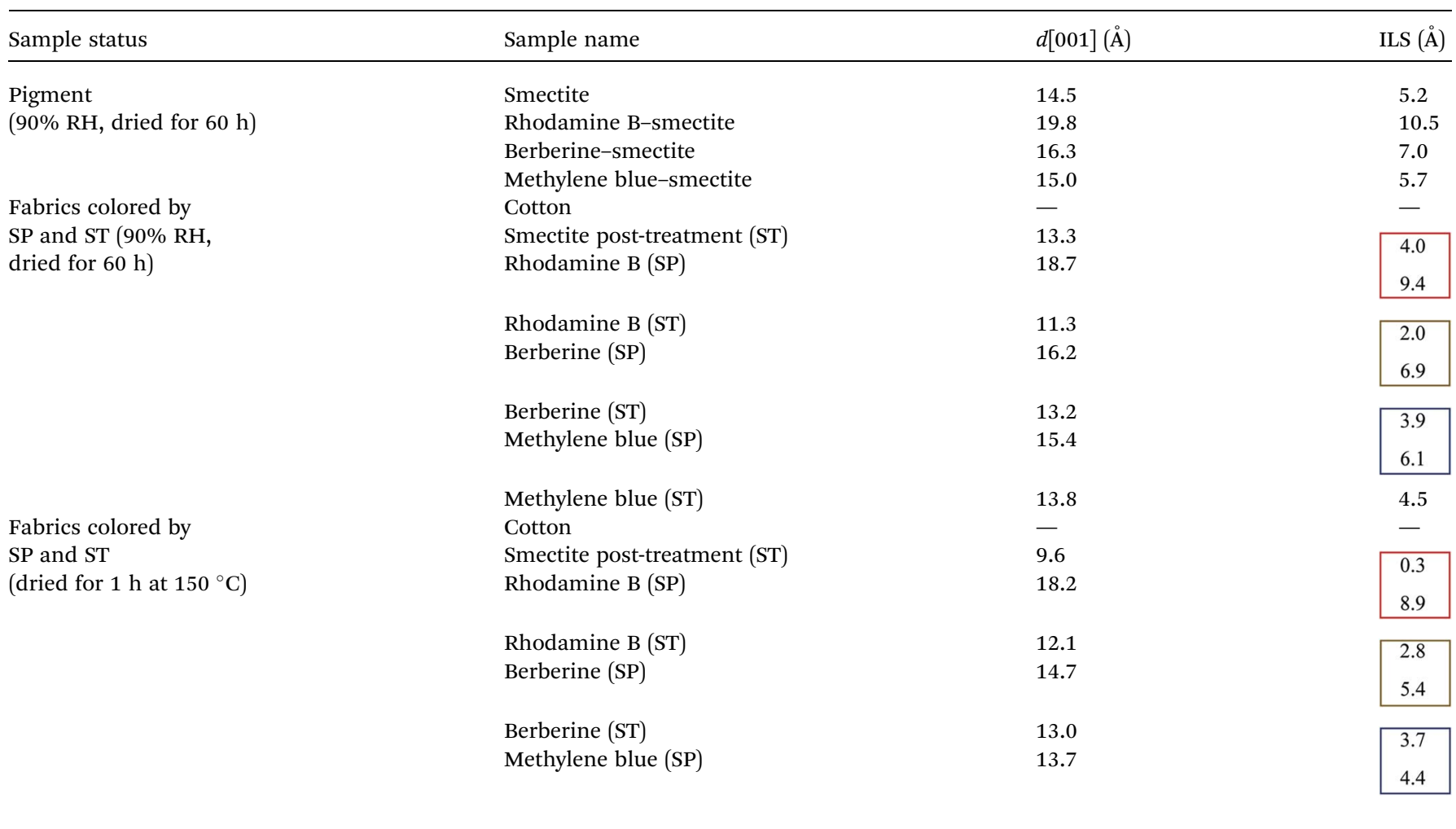




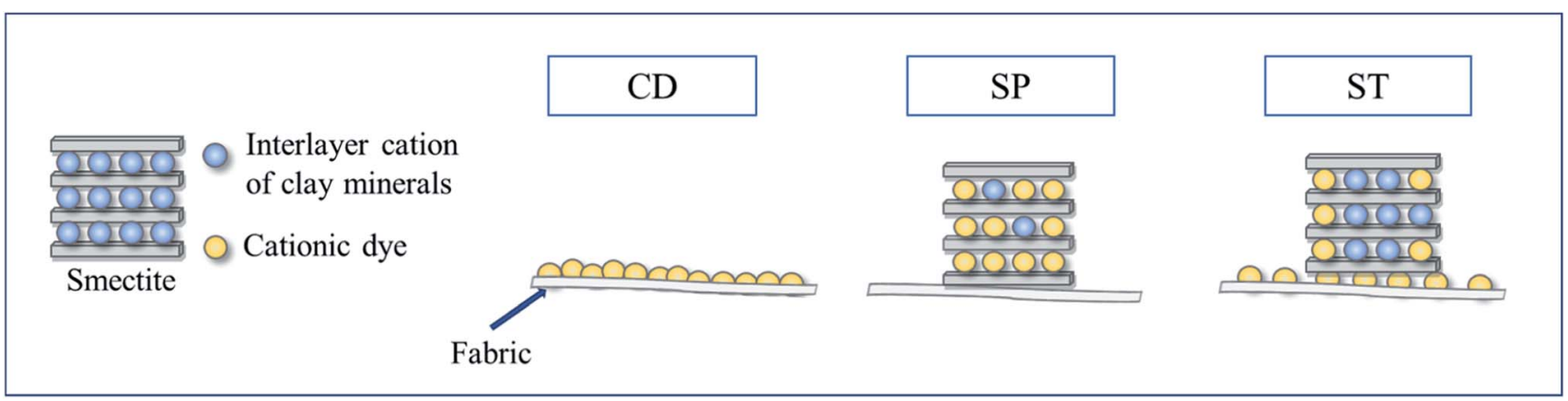

Scheme 3 Proposed mechanism by which SP and ST are colored on fabrics.

indicated that fabrics post-treated with smectite showed an ILS of only $0.3 \AA$, implying that only a trace amount of $\mathrm{H}_{2} \mathrm{O}$ remained. Even the samples colored using the different dyes showed no influence of the partially remaining $\mathrm{H}_{2} \mathrm{O}$, thereby allowing the ILS attributed to only the dyes to be identified more accurately. The ILS decreased in the order smectite pigment $\geq$ fabrics dyed by SP $>$ fabrics dyed by ST.

The proposed dyeing mechanism, as shown in Scheme 3, could be inferred from the results listed in Table 4. Assuming that the concentration of cations present in smectite is $100 \%$, it is predicted that smectite pigments prepared by ion exchange showed $90-100 \%$ dye intercalation, which adsorbed and adhered on the fabric surface as SP. Regarding ST, it is suspected that a portion of dye that had escaped from the dyed fabric during smectite post-treatment was simultaneously intercalated with the smectite coated on the dyed fabric surface.

\section{Conclusions}

Smectite pigments were prepared by intercalation of cationic dyes into smectite, as confirmed by XRD and TEM results. SP, which involved dyeing fabrics using only the prepared smectite pigments, showed very low dyeability and very small changes in washing fastness and light fastness. ST, which involved dyeing fabrics using cationic dyes and subsequent smectite posttreatment, showed good dyeability and washing fastness and light fastness superior to those in the case of $\mathrm{CD}$, which involved dying fabrics using CDs. In particular, ST was effective for dyeing cotton fabric, which has poor dyeability due to the non-affinity for CDs. When the dyeing mechanism was predicted by ILS measurements, ST showing superior dyeability and fastness was suspected to involve simultaneous intercalation of the cationic dye that escaped from the dyed fabric and fabric surface-coating. These the findings revealed that although smectite could be used to improve dyeability and fastness, more suitable dyeing conditions for smectite pigments and smectite post-treatment must be established, which will be a topic of future research.

\section{Conflicts of interest}

There are no conflicts of interest to declare.

\section{Acknowledgements}

This research was supported by Basic Science Research Program through the National Research Foundation of Korea (NRF) funded by the Ministry of Education (2016R1A6A1A03013422)

\section{Notes and references}

1 G. Crini, Bioresour. Technol., 2006, 97, 1061-1085.

2 J. Kyzioł-Komosińska, C. Rosik-Dulewska, M. Pająk, I. Krzyżewska and A. Dzieniszewska, Pol. J. Chem. Technol., 2014, 16, 33-40.

3 T. Robinson, B. Chandran and P. Nigam, Environ. Int., 2002, 28, 29-33.

4 V. K. Gupta and Suhas, J. Environ. Manage., 2009, 90, 23132342.

5 D. Shen, J. Fan, W. Zhou, B. Gao, Q. Yue and Q. Kang, J. Hazard. Mater., 2009, 172, 99-107.

6 Y. Ding and H. S. Freeman, Color. Technol., 2017, 133, 369375.

7 P. K. Malik, J. Hazard. Mater., 2004, B113, 81-88.

8 D. Cardon, Natural dyes: sources, tradition, technology and science, Taylor \& Francis, Ltd., London, 2007.

9 M. M. Kamel, F. Abdelghaffar and M. M. El-Zawahry, J. Nat. Fibers, 2011, 8, 289-307.

10 S. H. Lim and S. M. Hudson, J. Macromol. Sci., Polym. Rev., 2003, C43, 223-269.

11 K. Prabhu and M. Teli, J. Saudi Chem. Soc., 2014, 18, 864-872.

12 M. Shabbir, L. J. Rather, M. N. Bukhari, S. Ul-Islam, M. Shahid, M. A. Khan and F. Mohammad, J. Nat. Fibers, 2019, 16, 100-113.

13 M. Souissi, A. Guesmi and A. Moussa, J. Cleaner Prod., 2018, 204, 1143-1153.

14 J. S. Jung and M. Komaki, Sen'i Gakkaishi, 2011, 67, 66-71.

15 J. S. Jung, M. Komaki, T. Nakanishi, T. Satou and Y. Aikawa, Sen'i Gakkaishi, 2011, 67, 252-255.

16 T. Nakamura and M. Ogawa, Appl. Clay Sci., 2013, 83-84, 469-473.

17 E. Baez, N. Quazi, I. Ivanov and S. N. Bhattacharya, Adv. Powder Technol., 2009, 20, 267-272.

18 I. Chaaria, M. Fekib, M. Medhioubc, J. Bouzid, E. Fakhfakh and F. Jamoussi, J. Hazard. Mater., 2009, 172, 1623-1628. 
19 D. L. Guerra, W. L. L. Silva, H. C. P. Oliveira, R. R. Viana and C. Airoldi, J. Hazard. Mater., 2011, 186, 675-682.

20 R. E. Grim, Clay mineralogy, McGraw-Hill, 2nd edn, New York, 1968.

21 S. W. Bailey, Clays Clay Miner., 1980, 28, 73-78.

22 Reviews in mineralogy, vol. 19: hydrous phyllosilicates (exclusive of micas), ed. S.W. Bailey, Mineralogical Society of America, Chelsea, Michigan, 1988.

23 I. E. Odom, Philos. Trans. R. Soc. Lond. Ser. A Math. Phys. Sci., 1984, 311, 391-409.

24 F. Bergaya and G. Lagaly, Chapter 1-General introduction: clays, clay minerals and clay science, in Handbook of clay science, ed. F. Bergaya, B. K. G. Theng and G. Lagaly, Elsevier Science, 2nd edn, London, 2006.

25 Handbook of clay science, ed. F. Bergaya, G. Lagaly and B. K. G. Theng, Elsevier Science, 2nd edn, London, 2006.

26 The electron-optical investigation of clays, ed. J. A. Gard, Mineralogical Society of Great Britain and Ireland, London, 1971.

27 H. Van Olphen, An introduction to clay colloid chemistry. Interscience Publishers (Div. of John Wiley \& Sons), New York, 1963. 